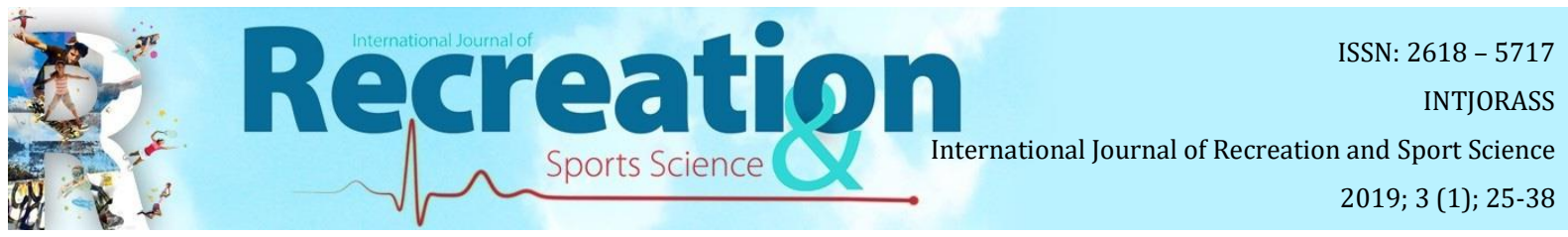

Research Article

\title{
A Study on the Effect of Personal Values on Leisure Motivation
}

\author{
Tebessüm AYYILDIZ DURHAN*
}

\begin{abstract}
The purpose of this study is to assess the effect of the personal values of licensed football players on leisure motivation, as well as to determine the relationship between personal values and leisure motivation. While the study group consisted of 208 footballer who continued to sport life in licensed football clubs in Ankara, While the study group consisted of 208 athletes who continued to sports life in different football clubs in Ankara, Personal Value Inventory (Asan et al., 2008) and Leisure Motivation Scale (Mutlu, 2008) were used for collecting data. The descriptive statistics, independent sample t test, Tukey (HSD-LSD) tests for analysis of differences between Anova groups, simple linear regression analysis and pearson correlation test were used. According to the findings obtained, the highest level of PV and LM were found in the highest PV score and the lowest in the respect and accuracy subdimension, while the lowest average was found in the discipline and responsibility subdimension. There was no significant relationship between participants' educational status, marital status, lack of leisure, availability of leisure and personal values and leisure motivation scales. In addition, there is a weak but positive direction between the personal values and the leisure motivation, which is found in favor of the participants with low income level in the perceived income variable, in favor of the longest-licensed players in the category of licensed players, correlation between the personal values and the leisure motivation as a result of the regression analysis revealed that there is a statistically significant positive linear relationship. As a result, it is concluded that personal value and leisure motivation levels are high, that certain variables affect this level, and that there is a weak but positive relationship between PV and LM. And it is the result of research that PV is the effect on LM.
\end{abstract}

Keywords: Value, Leisure, Personal value, Leisure motivation.

\section{Kişisel Değerlerin Boş Zaman Motivasyonu Üzerindeki Etkisine Yönelik Bir Araştırma}

\section{Öz}

$\mathrm{Bu}$ çalışmanın amacı lisanslı futbolcuların kişisel değerlerinin boş zaman motivasyonu üzerindeki etkisini değerlendirmek aynı zamanda kişisel değerler ve boş zaman motivasyonu arasındaki ilişkiyi belirlemektir. Çalışma grubunu Ankara ilinde farklı futbol kulüplerinde lisanslı olarak spor hayatına devam eden 208 sporcu oluştururken, verilerin toplanmasında Bireysel Değerler Envanteri (Asan ve diğ., 2008) ve Boş Zaman Motivasyon Ölçekleri (Mutlu, 2008) kullanılmıştır. Verilerin analizinde betimsel istatistiklerden, bağımsız örneklem t testinden, Anova gruplar arası farklılıkların analizi için Tukey (HSD-LSD) testlerinden, basit doğrusal regresyon analizinden ve pearson korelasyon testinden yararlanılmıştır. Elde edilen bulgulara göre KD ve BZM düzeylerinin yüksek olduğu, en yüksek KD puanının saygı ve doğruluk alt boyutunda elde ederken, en düşük ortalamayı disiplin ve sorumluk alt boyutunun aldığı görülmüştür. Katılımcıların eğitim durumu, medeni durum, boş zamanı yeterli bulup bulmama, verimli boş zamana sahip olma durumları ile kişisel değerler ve boş zaman motivasyon ölçekleri arasında anlamlı ilişki saptanamamıştır. Bunun yanı sıra katılımcıların yaş değişkeninde 18 yaş altı grup lehine, lisanslı sporcu olma kategorisinde en uzun süreyle lisansa sahip olan sporcuların lehine, algılanan gelir değişkeninde gelir düzeyini düşük olarak nitelendiren katılımcıların lehine anlamlı farklılıklar saptandı̆̆ 1 , kişisel değerler ve boş zaman motivasyonu arasında zayıf ancak pozitif yönlü ilişki olduğu, yapılan regresyon analizi sonucunda kişisel değerler ile boş zaman motivasyonunu arasında istatistiksel açıdan anlamlı pozitif doğrusal bir ilişki olduğu bulgular arasındadır. Sonuç olarak kişisel değerler ve boş zaman motivasyonu düzeyleri yüksek olduğu, belirli değişkenlerin bu düzeyi etkilediği, KD ve BZM arasında zayıf ancak pozitif yönlü ilişki olduğu, KD’nin BZM üzerinde etkisi olduğu araştırma sonucunu oluşturmaktadır.

Anahtar Kelimeler: Değer, Boş zaman, Kişisel değerler, Boş zaman motivasyonu.

* Gazi Üniversitesi, Spor Bilimleri Fakültesi, Ankara, Türkiye, tebessum@ gazi.edu.tr Orcid Id: https://orcid.org/0000-00032747-6933 


\section{INTRODUCTION}

Values defined as basic principles that guide human life play a key role in personal and social interactions as an important factor on mental processes. Man defines, interprets, refers and judges the world with his subjective perceptions as a bio-psycho-social being. It can be counted these values as an important factor that shapes these subjective perceptions and distinguishes a person's perception from the others. In addition to this, people know that values, norms, traditions, beliefs, anticipations and similar social components, which are accepted in the social environment they live in, learn from the developments and will increase the acceptability in society if they act accordingly (Çalışkur, 2010).

Value can be expressed as desire to serve individuals as a guiding principle in their lives (Karababa, 2014), and as a measure of the distinction that must be made to determine the significance of something.

Values are identified with people. In the process of socialization, values are learned and undertaken by individuals. In short, it integrates with one's personality (Aydin and Akyol Gürler, 2014). Values operate as a whole as an element with a central role in the mental world of the person, combining with the elements of viewpoints, evaluations, beliefs, motivations, attitudes, perceptions and emotions of the events and the expectations and norms of the society. Values shape the distinctive, peculiar synthesis of the individual in interaction with beliefs, attitudes, motives, needs, the norms of norms and anticipations. Thus, people who think differently, feel and behave are seen.

By overcoming specific situations, people can decide how they value their values (Çalışkur, 2010).
Akyol and Gürler (2014) summarize the characteristics of values as follows;

- Deep-rooted beliefs of historical accumulation

- It is assumed that the opinion has been reached

- Criteria used for decision making

- As a result of interacting with the environment, they gain functionality

- They form the substructure of attitudes

- They are motivating and encouraging

- They guide individuals to choose social roles

- Values affect individuals in determining purpose and direction

- Provides perspective on the person

Looking at the properties of the values, it seems to be a motivator. In this context, the concepts of motivation and motivation take place in the literature as descriptives used in the same sense. Motivation can be defined as an internal or external element that directs people towards a behavior. A recreational motivator may be a desire to develop football skills or to learn about the visual arts (Duran, 2017).

Motivation is associated with the state of the entity responsible for performing a specific activity at a particular time. The state of this organism is the result of a combination of internal (external) and external (environmental) factors as a rule (Konter, 1995). Inner and external factors combine behavior with personal values. Participation in leisure activities is also a combination of these factors.

Motivation to participate in activities in leisure is emphasized as an important structure in the sense of people's leisure 
behavior. It has been emphasized that with a good sense of motivation, one can gain a better sense of real behavior. Motivational structures determine stimuli for actual participation in a specific type of leisure. For example, it is likely that those who are physically motivated may participate in leisure activities with physical components (Choi and Fu, 2015; Gumus et al., 2019).

Considering that leisure is usually a free or voluntary time that individuals are left free of their job duties or responsibilities, the level of interest, motivation and participation in leisure activities can be determined by psycho-social measures, as in any complex human behavior. At this point, all of the biological, psychological, social and environmental factors contribute to leisure use and therefore leisure motivation (Reddon and Pope,1996; Gumus and Işik, 2018).

Most of the factors involved here are related to the individual attitude, thoughts, behavior and value structure of the individual. In this context, it is aimed to investigate the effect of individual values on leisure motivation in the context of the research, to determine the relationship between individual values and leisure motivation and to find out how certain variables affect individual values and leisure motivation.

\section{METHOD}

\section{Sample of the Study}

The study included 208 participants who played sports as licensed football players in Ankara and aimed sample method and general survey model were used because participants were asked to be licensed athletes.

\section{Data Collection Tools}

The Personal Values Inventory consisting of 47 items 5 sub-dimensions of the "Personal Values Inventory" scale developed by Roy (2003) by Asan et al. (2008) and the validity-reliance version of the Turkish form by Asan et al. (2008) is used in the survey. And the Leisure Motivation Scale (LMS) which is developed by Pelletier et al. (1991) and the validity reliability is done by Mutlu (2008) with 22 questions and 5 sub-dimensions were used in the study.

\section{Data Analysis}

Data were analyzed using descriptive statistics, Independent Sample T, One Way ANOVA, Tukey HSD and LSD tests, Simple Linear Regression Analysis and Pearson Correlation Analysis in intragroup comparisons. 


\section{FINDINGS}

Table 1. Percentage and frequency distributions for participants

\begin{tabular}{|c|c|c|c|}
\hline & \multicolumn{3}{|c|}{$\mathrm{N}=(208)$} \\
\hline & Variable & $\mathrm{f}$ & $\%$ \\
\hline \multirow{3}{*}{ Age } & $18<$ & 66 & 31,7 \\
\hline & $18-23$ & 99 & 47,6 \\
\hline & $24>$ & 43 & 20,7 \\
\hline \multirow{3}{*}{ Education level } & High school and low & 127 & 61,1 \\
\hline & Graduate and above & 81 & 38,9 \\
\hline & High & 38 & 18,3 \\
\hline \multirow[t]{2}{*}{ Percieved income } & Middle & 131 & 63,0 \\
\hline & Low & 39 & 18,8 \\
\hline \multirow{3}{*}{ Marital status } & Married & 33 & 15,9 \\
\hline & Single & 175 & 84,1 \\
\hline & Less than 3 years & 33 & 15,9 \\
\hline \multirow{3}{*}{ License duration } & $3-5$ years & 48 & 23,1 \\
\hline & $6-8$ years & 88 & 42,3 \\
\hline & 9 years and more & 39 & 18,8 \\
\hline \multirow{5}{*}{ Daily leisure } & None & 21 & 10,1 \\
\hline & Lower than 1-2 hours & 28 & 13,5 \\
\hline & $1-2$ hours & 55 & 26,4 \\
\hline & 3-4 hours & 56 & 26,9 \\
\hline & 5-6 hours & 48 & 23,1 \\
\hline \multirow{2}{*}{ Adequate leisure } & Inadequate & 115 & 55,3 \\
\hline & Adequate & 93 & 44,7 \\
\hline \multirow{2}{*}{ Sufficient leisure } & Yes & 121 & 58,2 \\
\hline & No & 87 & 41,8 \\
\hline
\end{tabular}

The participants of the study's study group were generally between the ages of $18-23$ $(47.6 \%)$, those with high school and below education level $(61.1 \%)$, those with middle income levels $(63.0 \%), 84.1 \%)$, those who had a license for 6-8 years $(42.3 \%)$, those who had 3 to 4 hours leisure per day (26.9\%), those who defined their leisure as inadequate $(55.3 \%)$, and they also expressed that they have spent their leisure $(58.2 \%)$ efficiently.

Table 2. Arithmetic mean and standard deviation values of personal values and LMS

\begin{tabular}{lcccc}
\hline & $\bar{x}$ & sd & Min & Max. \\
\cline { 2 - 5 } Personal values & 172,46 & 24,63 & 107,00 & 250,00 \\
\cline { 2 - 5 } Discipline and responsibility & 60,84 & 11,19 & 32,00 & 117,00 \\
\cline { 2 - 5 } Confidence and forgiveness & 41,44 & 7,19 & 24,00 & 67,00 \\
\cline { 2 - 5 } Honesty and sharing & 33,94 & 6,35 & 16,00 & 58,00 \\
Respect and accuracy & 21,39 & 3,91 & 11,00 & 30,00 \\
\hline Sharing and love & 15,26 & 2,96 & 5,00 & 20,00 \\
Leisure motivation & 72,59 & 11,60 & 42,00 & 122,00 \\
Motivationlessness & 8,56 & 2,54 & 3,00 & 15,00 \\
Knowing and achieving & 20,60 & 4,12 & 10,00 & 30,00 \\
\hline Stimulating life & 10,29 & 2,21 & 5,00 & 15,00 \\
\hline Identification & 20,88 & 5,14 & 12,00 & 67,00 \\
External regulation & 12,10 & 3,04 & 5,00 & 20,00 \\
\hline
\end{tabular}


The personal values and leisure motivation levels are high, the highest personal values score has the respect and accuracy subscale, and the lowest average discipline and responsibility subscale. The highest leisure motivation score is achieved by knowing and achieving the sub-dimension, while the lowest leisure motivation score is the identification sub-dimension.

Table 3. Anova test results between personal values and LMS and participants age variation

\begin{tabular}{|c|c|c|c|c|c|c|}
\hline & Age & $\mathbf{N}$ & $\bar{x}$ & sd & $\mathbf{F}$ & $\mathbf{p}$ \\
\hline Personal values & $\begin{array}{l}18< \\
18-23 \\
24> \\
\text { Total }\end{array}$ & $\begin{array}{c}66 \\
99 \\
43 \\
208\end{array}$ & $\begin{array}{l}174,65 \\
171,35 \\
171,65 \\
172,46\end{array}$ & $\begin{array}{l}26,68 \\
23,55 \\
24,16 \\
24,63\end{array}$ & 0,382 & 0,683 \\
\hline Discipline and responsibility & $\begin{array}{l}18< \\
18-23 \\
24> \\
\text { Total }\end{array}$ & $\begin{array}{c}66 \\
99 \\
43 \\
208\end{array}$ & $\begin{array}{l}60,53 \\
61,56 \\
59,65 \\
60,84\end{array}$ & $\begin{array}{l}10,97 \\
11,92 \\
9,817 \\
11,19\end{array}$ & 0,474 & 0,623 \\
\hline Confidence and forgiveness & $\begin{array}{l}18< \\
18-23 \\
24> \\
\text { Total }\end{array}$ & $\begin{array}{c}66 \\
99 \\
43 \\
208\end{array}$ & $\begin{array}{l}43,22 \\
40,31 \\
41,32 \\
41,44\end{array}$ & $\begin{array}{l}7,25 \\
6,80 \\
7,59 \\
7,19\end{array}$ & 3,329 & $0,038 *$ \\
\hline Honesty and sharing & $\begin{array}{l}18< \\
18-23 \\
24> \\
\text { Total }\end{array}$ & $\begin{array}{c}66 \\
99 \\
43 \\
208\end{array}$ & $\begin{array}{l}33,84 \\
33,86 \\
34,27 \\
33,94\end{array}$ & $\begin{array}{l}6,13 \\
6,30 \\
6,90 \\
6,35\end{array}$ & 0,074 & 0,929 \\
\hline Respect and accuracy & $\begin{array}{l}18< \\
18-23 \\
24> \\
\text { Total }\end{array}$ & $\begin{array}{c}66 \\
99 \\
43 \\
208\end{array}$ & $\begin{array}{l}21,77 \\
20,93 \\
21,88 \\
21,39\end{array}$ & $\begin{array}{l}4,19 \\
3,70 \\
3,93 \\
3,91\end{array}$ & 1,316 & 0,270 \\
\hline Sharing and love & $\begin{array}{l}18< \\
18-23 \\
24> \\
\text { Total }\end{array}$ & $\begin{array}{c}66 \\
99 \\
43 \\
208 \\
\end{array}$ & $\begin{array}{l}15,77 \\
15,29 \\
14,41 \\
15,26\end{array}$ & $\begin{array}{l}3,11 \\
2,68 \\
3,24 \\
2,96 \\
\end{array}$ & 2,763 & 0,065 \\
\hline Leisure motivation & $\begin{array}{l}18< \\
18-23 \\
24> \\
\text { Total }\end{array}$ & $\begin{array}{c}66 \\
99 \\
43 \\
208\end{array}$ & $\begin{array}{l}74,95 \\
72,89 \\
68,27 \\
72,59\end{array}$ & $\begin{array}{c}13,13 \\
11,64 \\
7,24 \\
11,60\end{array}$ & 4,519 & $0,012 *$ \\
\hline Motivationlessness & $\begin{array}{l}18< \\
18-23 \\
24> \\
\text { Total }\end{array}$ & $\begin{array}{c}66 \\
99 \\
43 \\
208\end{array}$ & $\begin{array}{l}9,28 \\
8,21 \\
8,27 \\
8,56\end{array}$ & $\begin{array}{l}2,48 \\
2,67 \\
2,11 \\
2,54\end{array}$ & 3,997 & $0,020 *$ \\
\hline Knowing and achieving & $\begin{array}{l}18< \\
18-23 \\
24> \\
\text { Total }\end{array}$ & $\begin{array}{c}66 \\
99 \\
43 \\
208\end{array}$ & $\begin{array}{l}21,16 \\
20,71 \\
19,46 \\
20,60\end{array}$ & $\begin{array}{l}4,49 \\
4,18 \\
3,14 \\
4,12\end{array}$ & 2,318 & 0,101 \\
\hline Stimulating life & $\begin{array}{l}18< \\
18-23 \\
24> \\
\text { Total }\end{array}$ & $\begin{array}{c}66 \\
99 \\
43 \\
208\end{array}$ & $\begin{array}{c}10,57 \\
10,40 \\
9,60 \\
10,29\end{array}$ & $\begin{array}{l}2,54 \\
2,00 \\
2,04 \\
2,21\end{array}$ & 2,782 & 0,064 \\
\hline Identification & $\begin{array}{l}18< \\
18-23 \\
24> \\
\text { Total }\end{array}$ & $\begin{array}{c}66 \\
99 \\
43 \\
208\end{array}$ & $\begin{array}{l}21,07 \\
21,49 \\
19,20 \\
20,88\end{array}$ & $\begin{array}{l}3,91 \\
6,34 \\
3,03 \\
5,14\end{array}$ & 3,080 & $0,048 *$ \\
\hline External regulation & $\begin{array}{l}18< \\
18-23 \\
24> \\
\text { Total }\end{array}$ & $\begin{array}{c}66 \\
99 \\
43 \\
208\end{array}$ & $\begin{array}{l}12,87 \\
11,85 \\
11,48 \\
12,10\end{array}$ & $\begin{array}{l}3,28 \\
3,04 \\
2,43 \\
3,04\end{array}$ & 3,414 & $0,035^{*}$ \\
\hline
\end{tabular}

According to the results of the ANOVA test between participants' age variable and personal values and leisure motivation scale, there was a significant difference between the confidence and forgiveness sub-dimension and the age variation of the participants. When the difference between participants who are under 18 years old and those who are between $18-23$ years is examined, it is observed that participants 
who are under 18 years old have a higher confidence and forgiveness sub-dimension than the other group. When the leisure motivation is considered, it is observed that there is a significant difference in favor of the group under the age of 18 in the difference between the groups under the age of 18 and the age group above the age of 24 years. When the significant differences in the motivationlessness, identification, and external regulation subscales of leisure motivation were examined, intergroup relations were analyzed. According to this, it was observed that motivation and external regulation subscale had a higher score of motivation in favor of the under 18 year old group between the under 18 and 18-23 age group. In the identification subscale, it is seen that subscale scores for the group between 18-23 age group and 24 years age group are higher in favor of the group between 18-23 age group.

Table 4. Anova test results between personal values and LMS and participants' license duration.

\begin{tabular}{|c|c|c|c|c|c|c|}
\hline & Duration of license & $\mathbf{N}$ & $\bar{x}$ & ss & $\mathbf{F}$ & $\mathbf{p}$ \\
\hline \multirow{5}{*}{ Personal values } & Less than 3 years & 33 & 165,63 & 23,67 & \multirow{5}{*}{1,374} & \multirow{5}{*}{0,252} \\
\hline & $3-5$ years & 48 & 171,56 & 22,53 & & \\
\hline & $6-8$ years & 88 & 173,47 & 26,21 & & \\
\hline & 9 years and more & 39 & 177,05 & 23,84 & & \\
\hline & Total & 208 & 172,46 & 24,63 & & \\
\hline \multirow{5}{*}{$\begin{array}{l}\text { Discipline and } \\
\text { responsibility }\end{array}$} & Less than 3 years & 33 & 58,39 & 10,14 & \multirow{5}{*}{1,026} & \multirow{5}{*}{0,382} \\
\hline & $3-5$ years & 48 & 59,85 & 10,45 & & \\
\hline & $6-8$ years & 88 & 61,64 & 12,30 & & \\
\hline & 9 years and more & 39 & 62,30 & 10,16 & & \\
\hline & Total & 208 & 60,84 & 11,19 & & \\
\hline \multirow{5}{*}{ Confidence and forgiveness } & Less than 3 years & 33 & 40,66 & 6,72 & \multirow{5}{*}{0,577} & \multirow{5}{*}{0,631} \\
\hline & $3-5$ years & 48 & 41,27 & 7,36 & & \\
\hline & $6-8$ years & 88 & 41,26 & 7,44 & & \\
\hline & 9 years and more & 39 & 42,74 & 6,89 & & \\
\hline & Total & 208 & 41,44 & 7,19 & & \\
\hline \multirow{5}{*}{ Honesty and sharing } & Less than 3 years & 33 & 31,69 & 6,46 & \multirow{5}{*}{1,783} & \multirow{5}{*}{0,151} \\
\hline & $3-5$ years & 48 & 33,89 & 5,39 & & \\
\hline & $6-8$ years & 88 & 34,54 & 6,78 & & \\
\hline & 9 years and more & 39 & 34,56 & 6,14 & & \\
\hline & Total & 208 & 33,94 & 6,35 & & \\
\hline \multirow{5}{*}{ Respect and accuracy } & Less than 3 years & 33 & 21,00 & 3,47 & \multirow{5}{*}{0,837} & \multirow{5}{*}{0,475} \\
\hline & $3-5$ years & 48 & 21,47 & 3,74 & & \\
\hline & $6-8$ years & 88 & 21,13 & 4,21 & & \\
\hline & 9 years and more & 39 & 22,23 & 3,77 & & \\
\hline & Total & 208 & 21,39 & 3,91 & & \\
\hline \multirow{5}{*}{ Sharing and love } & Less than 3 years & 33 & 14,06 & 2,91 & \multirow{5}{*}{2,709} & \multirow{5}{*}{$0,046^{*}$} \\
\hline & $3-5$ years & 48 & 15,31 & 3,06 & & \\
\hline & $6-8$ years & 88 & 15,36 & 3,01 & & \\
\hline & 9 years and more & 39 & 16,00 & 2,56 & & \\
\hline & Total & 208 & 15,26 & 2,96 & & \\
\hline \multirow{5}{*}{ Leisure motivation } & Less than 3 years & 33 & 73,21 & 12,06 & \multirow{5}{*}{0,785} & 0,503 \\
\hline & $3-5$ years & 48 & 74,27 & 13,38 & & \\
\hline & $6-8$ years & 88 & 71,23 & 10,21 & & \\
\hline & 9 years and more & 39 & 73,07 & 11,92 & & \\
\hline & Total & 208 & 72,59 & 11,60 & & \\
\hline \multirow{5}{*}{ Motivationlessness } & Less than 3 years & 33 & 9,00 & 2,52 & \multirow{5}{*}{0,530} & \multirow{5}{*}{0,662} \\
\hline & $3-5$ years & 48 & 8,68 & 2,59 & & \\
\hline & $6-8$ years & 88 & 8,37 & 2,57 & & \\
\hline & 9 years and more & 39 & 8,48 & 2,46 & & \\
\hline & Total & 208 & 8,56 & 2,54 & & \\
\hline
\end{tabular}


Ayyıldiz Durhan T. (2019). A Study on the Effect of Personal Values on Leisure Motivation. International Journal of Recreation and Sport Science, 3 (1); 25-38.

\begin{tabular}{|c|c|c|c|c|c|c|}
\hline \multirow{5}{*}{ Knowing and achieving } & Less than 3 years & 33 & 20,30 & 4,57 & \multirow{5}{*}{0,121} & \multirow{5}{*}{0,948} \\
\hline & $3-5$ years & 48 & 20,47 & 4,51 & & \\
\hline & $6-8$ years & 88 & 20,77 & 3,68 & & \\
\hline & 9 years and more & 39 & 20,61 & 4,32 & & \\
\hline & Total & 208 & 20,60 & 4,12 & & \\
\hline \multirow{5}{*}{ Stimulating life } & Less than 3 years & 33 & 10,21 & 2,11 & \multirow{5}{*}{0,804} & \multirow{5}{*}{0,493} \\
\hline & $3-5$ years & 48 & 10,62 & 2,48 & & \\
\hline & $6-8$ years & 88 & 10,05 & 2,12 & & \\
\hline & 9 years and more & 39 & 10,48 & 2,16 & & \\
\hline & Total & 208 & 10,29 & 2,21 & & \\
\hline \multirow{5}{*}{ Identification } & Less than 3 years & 33 & 20,69 & 3,31 & \multirow{5}{*}{1,097} & \multirow{5}{*}{0,351} \\
\hline & $3-5$ years & 48 & 22,02 & 7,76 & & \\
\hline & $6-8$ years & 88 & 20,36 & 4,47 & & \\
\hline & 9 years and more & 39 & 20,84 & 3,52 & & \\
\hline & Total & 208 & 20,88 & 5,14 & & \\
\hline \multirow{5}{*}{ External regulation } & Less than 3 years & 33 & 12,96 & 3,43 & \multirow{5}{*}{2,786} & \multirow{5}{*}{$0,042 *$} \\
\hline & $3-5$ years & 48 & 12,43 & 2,84 & & \\
\hline & $6-8$ years & 88 & 11,43 & 2,88 & & \\
\hline & 9 years and more & 39 & 12,48 & 3,08 & & \\
\hline & Total & 208 & 12,10 & 3,04 & & \\
\hline
\end{tabular}

There is a statistically meaningful difference in the sharing and love subdimension from the sub-dimensions of the personal values of the participants' license durations. According to this, it can be stated that the scores of sharing and love sub-dimension in favor of participants who say that they have 9 years and more license from participants who say that they have less than 3 years and 9 years and over have a license can be expressed. Regarding the leisure motivation scores, it is seen that the only significant difference in the external regulation is that the LSD test is less than 3 years after the test, and that the external regulation scores are favorable for footballers who are less licensed athletes for 3 years than the licensed footballers for 6-8 years. 
Table 5. Anova test results between personal values and LMS and income variance perceived by participants

\begin{tabular}{|c|c|c|c|c|c|c|}
\hline & Income & $\mathbf{N}$ & $\bar{x}$ & SS & $\mathbf{F}$ & $\mathbf{p}$ \\
\hline \multirow{4}{*}{ Personal values } & High & 38 & 168,44 & 23,29 & \multirow{4}{*}{0,615} & \multirow{4}{*}{0,541} \\
\hline & Middle & 131 & 173,39 & 26,34 & & \\
\hline & Low & 39 & 173,23 & 19,62 & & \\
\hline & Total & 208 & 172,46 & 24,63 & & \\
\hline \multirow{4}{*}{ Discipline and responsibility } & High & 38 & 58,76 & 9,26 & \multirow{4}{*}{0,834} & \multirow{4}{*}{0,436} \\
\hline & Middle & 131 & 61,42 & 12,02 & & \\
\hline & Low & 39 & 60,89 & 9,93 & & \\
\hline & Total & 208 & 60,84 & 11,19 & & \\
\hline \multirow{4}{*}{ Confidence and forgiveness } & High & 38 & 42,60 & 8,50 & \multirow{4}{*}{0,601} & \multirow{4}{*}{0,549} \\
\hline & Middle & 131 & 41,17 & 7,09 & & \\
\hline & Low & 39 & 41,23 & 6,13 & & \\
\hline & Total & 208 & 41,44 & 7,19 & & \\
\hline \multirow{4}{*}{ Honesty and sharing } & High & 38 & 31,50 & 5,86 & \multirow{4}{*}{3,703} & \multirow{4}{*}{$0,026^{*}$} \\
\hline & Middle & 131 & 34,34 & 6,78 & & \\
\hline & Low & 39 & 35,00 & 4,59 & & \\
\hline & Total & 208 & 33,94 & 6,35 & & \\
\hline & High & 38 & 20,97 & 3,94 & \multirow{4}{*}{0,272} & \multirow{4}{*}{0,762} \\
\hline \multirow{4}{*}{ Respect and accuracy } & Middle & 131 & 21,49 & 3,98 & & \\
\hline & Low & 39 & 21,48 & 3,70 & & \\
\hline & Total & 208 & 21,39 & 3,91 & & \\
\hline & High & 38 & 14,50 & 2,96 & \multirow{4}{*}{1,618} & \multirow{4}{*}{0,201} \\
\hline \multirow{3}{*}{ Sharing and love } & Middle & 131 & 15,48 & 3,03 & & \\
\hline & Low & 39 & 15,28 & 2,68 & & \\
\hline & Total & 208 & 15,26 & 2,96 & & \\
\hline \multirow{4}{*}{ Leisure motivation } & High & 38 & 72,07 & 13,31 & \multirow{4}{*}{0,082} & \multirow{4}{*}{0,921} \\
\hline & Middle & 131 & 72,58 & 11,55 & & \\
\hline & Low & 39 & 73,15 & 10,18 & & \\
\hline & Total & 208 & 72,59 & 11,60 & & \\
\hline & High & 38 & 9,15 & 2,68 & & \\
\hline & Middle & 131 & 8,45 & 2,48 & & \\
\hline Motivationlessness & Low & 39 & 8,38 & 2,59 & 1,265 & 0,284 \\
\hline & Total & 208 & 8,56 & 2,54 & & \\
\hline & High & 38 & 19,73 & 3,86 & & \\
\hline & Middle & 131 & 20,78 & 4,29 & & \\
\hline Knowing and achieving & Low & 39 & 20,82 & 3,76 & 1,021 & 0,362 \\
\hline & Total & 208 & 20,60 & 4,12 & & \\
\hline & High & 38 & 10,07 & 2,27 & & \\
\hline & Middle & 131 & 10,38 & 2,25 & & \\
\hline Stimulating life & Low & 39 & 10,17 & 2,06 & 0,350 & 0,705 \\
\hline & Total & 208 & 10,29 & 2,21 & & \\
\hline & High & 38 & 20,92 & 8,22 & & \\
\hline & Middle & 131 & 20,93 & 4,48 & & \\
\hline Identification & Low & 39 & 20,69 & 3,08 & 0,035 & 0,966 \\
\hline & Total & 208 & 20,88 & 5,14 & & \\
\hline & High & 38 & 12,05 & 3,36 & & \\
\hline & Middle & 131 & 12,03 & 3,02 & & \\
\hline External regulation & Low & 39 & 12,38 & 2,83 & 0,200 & 0,819 \\
\hline & Total & 208 & 12,10 & 3,04 & & \\
\hline
\end{tabular}

$* \mathrm{p}<0.05$ is statistically significant.

When we examine Table 5, we observe that the level of honesty and sharing in the subscale of personal values scale shows that there is a significant difference between all the groups in the honesty and sharing sub-dimension. It is seen that there is no significant difference in the total scores of leisure motivation and subscale scores. 
Table 6. Results of correlation analysis between personal values and leisure motivation scale

\begin{tabular}{|c|c|c|c|c|c|c|c|c|c|c|c|c|}
\hline & & 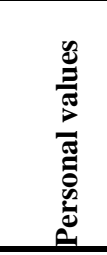 & 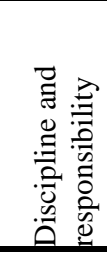 & 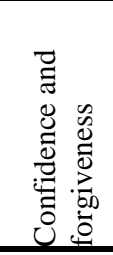 & 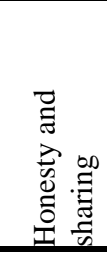 & 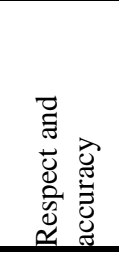 & 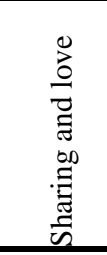 & 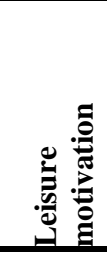 & 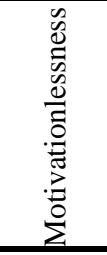 & 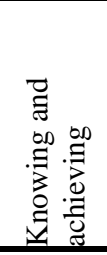 & 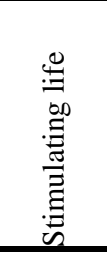 & 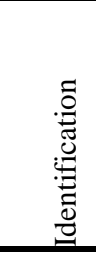 \\
\hline $\begin{array}{l}\text { Discipline and } \\
\text { responsibility }\end{array}$ & $\begin{array}{l}\text { Pearson C. } \\
\text { Sig. }\end{array}$ & $\begin{array}{l}, 883 * * \\
, 000 \\
\end{array}$ & 1 & & & & & & & & & \\
\hline $\begin{array}{l}\text { Confidence and } \\
\text { forgiveness }\end{array}$ & $\begin{array}{l}\text { Pearson C. } \\
\text { Sig. }\end{array}$ & $\begin{array}{l}, 709 * * \\
, 000\end{array}$ & $\begin{array}{l}, 452 * * \\
, 000\end{array}$ & 1 & & & & & & & & \\
\hline $\begin{array}{l}\text { Honesty and } \\
\text { sharing }\end{array}$ & $\begin{array}{l}\text { Pearson C. } \\
\text { Sig. }\end{array}$ & $\begin{array}{l}, 818 * * \\
, 000\end{array}$ & $\begin{array}{l}, 689 * * \\
, 000\end{array}$ & $\begin{array}{l}, 387 * * \\
, 000\end{array}$ & 1 & & & & & & & \\
\hline $\begin{array}{l}\text { Respect and } \\
\text { accuracy }\end{array}$ & $\begin{array}{l}\text { Pearson C. } \\
\text { Sig. }\end{array}$ & $\begin{array}{l}, 729 * * \\
, 000 \\
\end{array}$ & $\begin{array}{l}, 499 * * \\
, 000 \\
\end{array}$ & $\begin{array}{l}, 470 * * \\
, 000 \\
\end{array}$ & $\begin{array}{l}, 585 * * \\
, 000 \\
\end{array}$ & 1 & & & & & & \\
\hline $\begin{array}{l}\text { Sharing and } \\
\text { love }\end{array}$ & $\begin{array}{l}\text { Pearson C. } \\
\text { Sig. }\end{array}$ & $\begin{array}{l}, 625^{* *} \\
, 000 \\
\end{array}$ & $\begin{array}{l}, 527 * * \\
, 000\end{array}$ & $\begin{array}{l}, 300 * * \\
, 000\end{array}$ & $\begin{array}{l}, 460 * * \\
, 000\end{array}$ & $\begin{array}{l}, 451 * * \\
, 000\end{array}$ & 1 & & & & & \\
\hline $\begin{array}{l}\text { Leisure } \\
\text { motivation }\end{array}$ & $\begin{array}{l}\text { Pearson C. } \\
\text { Sig. }\end{array}$ & $\begin{array}{l}, 222 * * \\
, 001\end{array}$ & $\begin{array}{l}, 165 * * \\
, 017\end{array}$ & $\begin{array}{l}, 193 * * \\
, 005\end{array}$ & $\begin{array}{l}, 157 * \\
, 023\end{array}$ & $\begin{array}{l}, 195^{*} \\
, 005\end{array}$ & $\begin{array}{l}, 194 * * \\
, 005\end{array}$ & 1 & & & & \\
\hline $\begin{array}{l}\text { Motivationlessn } \\
\text { ess }\end{array}$ & $\begin{array}{l}\text { Pearson C. } \\
\text { Sig. }\end{array}$ & $\begin{array}{l}, 100 \\
150 \\
\end{array}$ & $\begin{array}{l}-, 012 \\
, 864 \\
\end{array}$ & $\begin{array}{l}, 273 * * \\
, 000 \\
\end{array}$ & $\begin{array}{l}, 004 \\
, 958 \\
\end{array}$ & $\begin{array}{l}140^{*} \\
, 044\end{array}$ & $\begin{array}{l}-, 030 \\
, 665 \\
\end{array}$ & $\begin{array}{l}, 497 * * \\
, 000\end{array}$ & 1 & & & \\
\hline $\begin{array}{l}\text { Knowing and } \\
\text { achieving }\end{array}$ & $\begin{array}{l}\text { Pearson C. } \\
\text { Sig. }\end{array}$ & $\begin{array}{l}\text {,231** } \\
, 001 \\
\end{array}$ & $\begin{array}{l}, 242 * * \\
, 000\end{array}$ & $\begin{array}{l}, 085 \\
, 223 \\
\end{array}$ & $\begin{array}{l}, 205 * * \\
, 003 \\
\end{array}$ & $\begin{array}{l}, 156^{*} \\
, 024 \\
\end{array}$ & $\begin{array}{l}, 202 * * \\
, 003\end{array}$ & $\begin{array}{l}, 765 * * \\
, 000 \\
\end{array}$ & $\begin{array}{l}153^{*} \\
, 028 \\
\end{array}$ & 1 & & \\
\hline Stimulating life & $\begin{array}{l}\text { Pearson C. } \\
\text { Sig. }\end{array}$ & $\begin{array}{l}202^{* *} \\
, 003 \\
\end{array}$ & $\begin{array}{l}, 163^{*} \\
, 019 \\
\end{array}$ & $\begin{array}{l}146^{*} \\
, 036 \\
\end{array}$ & $\begin{array}{l}146^{*} \\
, 035 \\
\end{array}$ & $\begin{array}{l}, 152^{*} \\
, 028 \\
\end{array}$ & $\begin{array}{l}, 216 * * \\
, 002 \\
\end{array}$ & $\begin{array}{l}, 636 * * \\
, 000 \\
\end{array}$ & $\begin{array}{l}129 \\
, 064 \\
\end{array}$ & $\begin{array}{l}, 554 * * \\
, 000\end{array}$ & 1 & \\
\hline Identification & $\begin{array}{l}\text { Pearson C. } \\
\text { Sig. }\end{array}$ & $\begin{array}{l}, 106 \\
, 129 \\
\end{array}$ & $\begin{array}{l}, 088 \\
, 205 \\
\end{array}$ & $\begin{array}{l}, 089 \\
, 199 \\
\end{array}$ & $\begin{array}{l}, 050 \\
, 477 \\
\end{array}$ & $\begin{array}{l}, 080 \\
, 249 \\
\end{array}$ & $\begin{array}{l}, 171^{*} \\
, 014 \\
\end{array}$ & $\begin{array}{l}, 768 * * \\
, 000 \\
\end{array}$ & $\begin{array}{l}, 210 * * \\
, 002 \\
\end{array}$ & $\begin{array}{l}, 404 * * \\
, 000 \\
\end{array}$ & $\begin{array}{l}, 359 * * \\
, 000 \\
\end{array}$ & 1 \\
\hline $\begin{array}{l}\text { External } \\
\text { regulation }\end{array}$ & $\begin{array}{l}\text { Pearson C. } \\
\text { Sig. }\end{array}$ & $\begin{array}{l}, 139 * \\
, 045\end{array}$ & $\begin{array}{l}, 043 * \\
, 540\end{array}$ & $\begin{array}{l}, 173 * \\
, 013\end{array}$ & $\begin{array}{l}, 101 \\
, 145\end{array}$ & $\begin{array}{l}, 223 * * \\
, 001\end{array}$ & $\begin{array}{l}, 064 \\
, 357\end{array}$ & $\begin{array}{l}, 588 * * \\
, 000\end{array}$ & $\begin{array}{l}, 472 * * \\
, 000\end{array}$ & $\begin{array}{l}, 317 * * \\
, 000\end{array}$ & $\begin{array}{l}, 173 * \\
, 013\end{array}$ & $\begin{array}{l}, 264 * * \\
, 000\end{array}$ \\
\hline
\end{tabular}

There was a statistically weak (0.20-0.39)

between personal values and leisure (Alpar, 2014: 356) but positive relationship motivation.

Table 7. Regression analysis results between personal values and leisure motivation scales

\begin{tabular}{|c|c|c|c|c|c|c|}
\hline & & B & Std. Error & ß & $\mathrm{t}$ & $\mathrm{p}$ \\
\hline \multirow[t]{2}{*}{ Leisure motivation } & & 138,200 & 10,601 & & 13,037 & $0,000^{*}$ \\
\hline & & 0,472 & 0,144 & 0,222 & 3,273 & 0,001 \\
\hline $\begin{array}{l}\mathrm{R}=0,22 \\
\mathrm{~F}=10,711\end{array}$ & $\begin{array}{r}\mathrm{R}^{2}=0,049 \\
\mathrm{p}<0,001\end{array}$ & & & & & \\
\hline
\end{tabular}

Dependent variable: personal values

There is a statistically significant positive linear relationship between personal values and leisure motivation. Personal values, which is dependent variable, can be said to be effective in explaining the change in leisure motivation, which is an independent variable. 


\section{DISCUSSION AND CONCLUSION}

According to the findings of the research, it was observed that the PV and LM levels were higher, the highest PV score was the respect and accuracy subscale, while the lowest mean was the discipline and responsibility subscale. There was no significant relationship between participants' educational status, marital status, lack of leisure, availability of leisure and personal values and leisure motivation scales.

In addition, there is a weak but positive direction between the personal values and the leisure motivation, which is found in favor of the participants with low income level in the perceived income variable, in favor of the longest-licensed athlete in the category of licensed athlete, correlation between the personal values and the leisure motivation as a result of the regression analysis revealed that there is a statistically significant positive linear relationship. As a result, it is concluded that personal variables and leisure motivation levels are high, that certain variables affect this level, and that there is a weak but positive relationship between $\mathrm{PV}$ and $\mathrm{LM}$, the effect of PV on LM.

Erdogan carried out a similar study in our research in 2014 and analyzed the individual values in professional soccer players. In his research, he found statistically significant relationships in the survey. The educational status, which league playing at, the year of professionalism, the national team playing situation, the football playing situation abroad, the replacement status, and the longest duty cycle status variables in a club have significant differences. On the contrary to our research it is seen that there are significant differences in marital situation in favor of single participants, in the discipline and responsibility subdimension in favor of participants with low education level.

Since our research group is made up of soccer players, it is not a matter of looking for a meaningful difference with the gender variable. In this sense, there are also studies describing the relationship between gender and personal values in the field. Uyguç (2003) aimed to reveal the gender and faculty differences in the importance that the students gave to the values. When the results were examined, it was found that there was no meaningful difference according to the faculty differences while there were meaningful differences according to the gender.

There are many studies that centered on individual values and have contributed to the literature on research on reflection in different fields (Muller, 1989; Slimak and Dietz, 2006; Rokeach, 1972; Yarar, 2016). When we look at other works related to personal values, personal values are examined by self-actualization and selftranscendence. The research hypotheses are based on whether the values of selfactualization and self-transcendence of employees are high or low.

The most important finding is that the efficacy of distribution and procedural justice on pride is higher in individuals who are both self-transcendent and highly self-fulfilling (Çınar Altıntaş, 2006). In another study, it was aimed to determine the extent to which the personal values of the classroom teacher candidates predicted their attitudes toward teaching, and it was revealed that the personal values of the teacher candidates predicted their attitudes towards the teaching profession (Bektaş and Nalçacı, 2012). 
In the literature, it is seen that there are no studies investigating the relationship between leisure motivation and personal values with comparison measures such as organizational commitment, occupational satisfaction and life satisfaction. It is seen that research literature for comparing personal values and leisure motivation with different measurement tools appears to be included. One of the present ones;As a result of the study conducted by A ğduman (2014) with the participation of the students studying at different sections of Atatürk University, leisure motivation and leisure satisfaction were related to each other and it was found that this relationship was positive in parallel with our research. Similarly, Beggs and Elkins (2010) found a positive relationship between leisure motivation and leisure satisfaction in their research.

In the dissertation of Güler in 2017, studies were conducted in order to determine the levels of leisure motivation levels of university students, to compare them in terms of demographic variables, and to determine the relations between them. when the results were examined, it was found that the dimensions of achievement and knowing of high idle-time motivation levels were more prominent. In contrast to our research, there was no relationship between leisure and leisure motivation levels, but there are significant differences between age variable and LM in parallel with our research.

Lloyd and others have put forward a study that in 2007 leisure motivation could help heal. In another study conducted on the elderly, leisure motivation was found to predict life satisfaction (Guinn, 2009). Another study was conducted with 140 female and 290 male participants to determine the relationship between academic achievement and leisure motivation of physical education students. According to the results, statistically significant differences were found between the subscales of the academic achievement scale and the leisure motivation subdimensions. These; knowing and achieving, stimulating life and external regulation. There was a positive correlation between knowledge and achievement subscale and identification / subscale subscale and academic achievement scale. While a positive relationship was found between stimulating life and academic achievement sub-dimension, it was observed that the level of academic motivation increased with stimulation level of life. A positive correlation was found between the identity / inference subscale and the academic achievement scale. Another finding is that there is a weak relationship between the academic motivation scale and the leisure motivation scale subscale to the external regulation subscale. As the level of academic motivation of physical education students decreases, the motivation of external regulation decreases accordingly (Çalık et al., 2014).

In a survey conducted by Yerlisu Lapa and others on 166 male participants in Kayseri in 2012, physical education and sports teachers, it is found that there is positive linear relationship between life satisfaction, leisure motivation and participation frequency to leisure acvitivites. There was no significant difference in social and artistic/cultural activities with respect to active/passive participation, whereas gender had a significant difference in frequency of participation in leisure activities and in leisure motivation according to active/passive participation in sporting activities. 
Akyüz and others (2018) studied the effects of leisure motivation on the level of happiness of students who are studying in the recreation section according to their demographic variables, there was no significant relationship between the mean scores of the LM and the happiness scale and no significant difference was found between the age and income variables and the reverse of our research. However, there is a moderately positive relationship between happiness and leisure motivation in the sub-dimensions of knowing and achieving, stimulating and living; a negative correlation was found in the subdimensions of identification, inversion, and external regulation. As a result of multiple linear regression analysis; motivation, knowing and achieving, stimulus and life, identification, external regulation and gender variables together have a significant effect on happiness $(\mathrm{R}=$ $0,561 ; \mathrm{R} 2=0,315), \mathrm{F}(6-143)=10,945$; $\mathrm{p}<0,05)$. When the significance tests of the regression coefficients are taken into consideration, it is seen that only the knowing and achieving of the predictive variables and the subscales of leisure motivation to survive and stimulate life are significant predictors of happiness.

In another study conducted with 222 male and 309 female participants, a crosssectional analysis of the motivations for participation in physical activity in the adult life stages was conducted. There was no significant difference when gender was evaluated. A significant difference was found in the ANOVA test for life stages. Individuals between the ages of 18-24 were found to have high motivation. In addition to this, it has been observed that over 65 participants have high motivation due to the socialization that leisure brings (Beggs et al., 2014).
In the study on secondary school students in the United States, it was aimed to examine the motivation and behavioral support of the parents of the students and the physical education teachers on leisure activities. It is seen that parents play a statistically significant role on leisure motivation. The motivation of the adolescents for their leisure activities was recorded as the most important factor in determining the behavior of leisure activities (McDavid, Cox e Amorose, 2012; Gumus and Karakullukcu, 2015). In another study, it was aimed to examine the motivation of sports education programs in high schools for physical education and leisure activities, and 568 high school students participated in the research. It was held within the scope of a 2-year sports training program. The results suggest that the motivation for leisure activities is limited to the students through sports programs. Within the scope of the sports program, the students applied volleyball, badminton, handball, football, basketball branches (Wallhead, Garn and Vidoni, 2014; Ayar and Koç,2018).

As a result, it was concluded that personal values and leisure motivation levels were high for this study group, that personal values had an effect on leisure motivation, there was a significant positive correlation between two components, and that there were significant differences between the two variables. Personal values and leisure motivation can be evaluated on different sample groups and psycho-social studies are suggested to expand the literature.

\section{REFERENCES}

Ağduman, F. (2014). Study on Leisure Motivations and Satisfactions of University Students. Atatürk 
University Institute of Health Science, Master Thesis.

Akyüz, H., Yaşartürk, F., Karataş, İ., Türkmen, M., \& Zorba, E., (2018) The effect of free time motivations on happiness level of students in recreation department Journal of Human Sciences, 15(2), 1086-1096.

Alpar, R. (2014). Spor, Sağlık ve Eğitim Bilimlerinden Örneklerle Uygulamalı İstatistik ve GeçerlikGüvenirlik-SPSS'de Çözümleme Adımları İle Birlikte. Yenilenmiş 3. Bask1, Ankara: Detay Yayinc1lik,356.

Asan, T., Ekşi, F., Doğan, A. \& Ekşi, H. (2008). Bireysel değerler envanteri'nin dilsel eşdeğerlik geçerlik ve güvenirlik çalışması. Marmara Üniversitesi Atatürk Eğitim Fakültesi Eğitim Bilimleri Dergisi, 27, 15-38.

Ayar, H. \& Koç, M.C. (2018). 1980-2018 Yillari Arasi Türkiye'de Rekreasyon Alaninda Yapilan Lisansüstü Tezlerin İncelenmesi. Sportif Baklş: Spor ve Eğitim Bilimleri Dergisi, 96107.

Aydın, M. Z., Akyol Gürler, Ş. (2014). Okulda Değerler Eğitimi:Yöntemler Etkinlikler Kaynaklar 4. Basım. Ankara: Nobel Yayıncılık,1-4.

Beggs, B. A., Kleparski,T., Elkins,D. \& Hurd, A. (2014). Leisure Motivation of Older Adults in Relation to Other Adult Life Stages. Activities, Adaptation \& Aging, 38:3, 175-187.

Beggs, B. A. \& Elkins, D. J. (2010). The Influence of Leisure Motivation on Leisure Satisfaction. LARNet - The Cyber Journal of Applied Leisure and Recreation Research, 36.

Bektaş, F. \& Nalçacı, A. (2012). The relationship between personal values and attitude towards teaching profession. Educational Sciences: Theory \& Practice, 12(2), Supplementary Special Issue, Spring, 1244-1248.

Çalık, F., Gülle, M., Sertbaş, K., Sarı, İ. \& Kaya, E. (2014). Analysis and examination of the relationship between leisure motivation andacademic motivation of students of schools of physical education and sports. Journal of Physical Education and Sport (JPES), 14 (1) $127-133$.

Çalışkur, A. (2010). Psikoljide Değerler ve Gençlik. İstanbul: Papatya Yayıncılık, 9-22.

Choi, S. \& Fu, X. (2015). Re-examining the Dimensionality of Leisure Motivation and Leisure Satisfaction in a Multicultural Context: Evidence from Macau. Humanities \& Social Sciences Reviews(HSSR).3(1),6-10.

Çınar Altıntaş, F. (2006). Bireysel değerlerin örgütsel adalet ve sonuçları ilişkisinde yönlendirici etkisi: akademik personel üzerinde bir analiz. İşletme Fakültesi Dergisi, 7 (2), 19-40.

Duran, N. (2017). Leisure Motivation. http://samples.jbpub.com/97812841 06817/9781284106879_CH02_Print .pdf

Erdoğan, B. S. (2014). A study on personal values of proffesional footbol players. Gazi University Graduate School of Educational Sciences, Ph. D. Thesis.

Guinn, B. (2009). Leisure Behavior Motivation and the Life Satisfaction of Retired Persons. Activities, Adaptation \& Aging, 23:4, 13-20.

Güler, H. (2017). Investigation of the Effects of Leisure Time Constraints of the University Students in Physical Education and Sport Scholls on their Leisure Time 
Motivation: Bartın University Sample. Bartın University, Institute of Educational Sciences Department of Physical Education and Sports Teaching, Master Thesis.

Gumus, H., \& Isik, O. (2018). The Relationship of Physical Activity Level, Leisure Motivation and Quality of Life in Candidate Teachers. International Journal of Progressive Education, 14(5), 22-32.

Gümüş, H., \& Karakullukçu, Ö. F. (2015). Leisure satisfaction in football and basketball fans: a sample of Afyonkarahisar. International Journal of Science Culture and Sport (IntJSCS)(3), 401-409.

Gumus, H., Honca, A. A., \& Cetinkaya, T. (2019). Perceived Social Support in Recreational Activity Participation: A Study on Students. Higher Education Studies, 9(1), 151-158.

Karababa, A. (2014). Değer Olgusu. B. Dilmaç, H. H. Bircan (Editörler) Değerler ve Değerler Psikolojisi. Ankara: Pegem Akademi, 2.

Konter, E. (1995). Sporda Motivasyon. Saray İzmir:Medikal Yayıncılık,9.

Lloyd, C., King, R., McCarthy, M., Scanlan, M. (2007). The association between leisure motivation and recovery: A pilot study. Australian Occupational Therapy Journal, 54, 33-41.

McDavid, L., Cox, A. E. \& Amorose, A. J. (2012).The relative roles of physical education teachers and parents in adolescents' leisure-time physical activity motivation and behavior. Psychology of Sport and Exercise. 13, 99-107.

Mutlu, İ. (2008). Egzersiz Yapan Kişilerin Boş Zamanlarına Yönelik Tutumları Üzerine Bir Araştırma (Kayseri İli Örneği), Niğde University, Institute of Social Sciences, Unpublished Master Thesis.

Pelletier, L.G., Vallerand, R.J., Blais, M.R. \& Brière, N.M. (1991). "Leisure Motivation Scale (LMS-28)", http://www.er.uqam.ca/nobel/r2671 0/LRCS/echelles_en.htm.

Reddon, J. R., Pope, G. A., Friel, J. P., \& Sinha, B. K. (1996). Leisure motivation in relation to psychosocial adjustment and personality in young offender and high school samples. Journal of Clinical Psychology, 52(6), 679-685.

Rokeach, M. (1972). Beliefs, attitudes, and values: A theory of organization and change. San Francisco, CA: JosseyBass Inc.

Roy, A. (2003). Factor analysis and initial validation of the personal values inventory. Unpublished doctorate dissertation, Tennessee State University, USA.

Uyguç, N. (2003). Gender, personal values and choice of profession. Journal of D.E.Ü.İ.İ.B.F., 18(1), 93-103.

Wallhead, T. L., Garn, A. C. \& Vidoni, C. (2014). Effect of a Sport Education Program on Motivation for Physical Education and Leisure-Time Physical Activity. Research Quarterly for Exercise and Sport, 85, 478-487.

Yarar, B. (2016). The relationship between personal values, calling and work engagement. Marmara Üniversitesi Sosyal Bilimler Enstitüsü, Yüksek Lisans Tezi.

Yerlisu Lapa, T., Ağyar, E. \& Bahadır, Z. (2012). Life satisfaction, leisure motivation, leisure participaton: An analysis on physical education and sport teachers (Kayseri city example) Spormetre Beden Eğitimi ve Spor Bilimleri Dergisi, 2012, 10(2) 53-59. 